\title{
Validation of multi-dimensional force measurement using zero-compliance mechanism
}

\author{
Md Helal An NAHIYAN*, Takeshi MIZUNO*, Masaya TAKASAKI*, Yuji ISHINO**, \\ Masayuki HARA* and Daisuke YAMAGUCHI* \\ *Graduate School of Science and Engineering, Saitama University \\ Shimo-Okubo 255, Sakura-ku, Saitama 338-8570, Japan \\ E-mail: nahiyan.m.h.a.130@ms.saitama-u.ac.jp \\ ${ }^{* *}$ General Technical Support Centre, Saitama University \\ Shimo-Okubo 255, Sakura-ku, Saitama 338-8570, Japan
}

Received: 28 December 2017; Revised: 28 February 2018; Accepted: 26 March 2018

\begin{abstract}
A novel force measurement instrument is designed and fabricated to validate multi-dimensional force measurement using zero compliance mechanism. In each axis, there is a point of force and a detection point for zero-compliance force measurement. Both the detection point and the point of force are suspended in series and attached with the base frame through leaf springs. When external force is applied at the point of force, it moves from its original position. The motion of the point of force can be regulated by displacing the detection point in the same or opposite direction of the applied force. The control system is designed and implemented to control the movement of the point of force and the detection point in vertical and horizontal direction force measurement. The effect of applied force on the point of force is cancelled to maintain its position by displacing the detection point. The motion of the detection point is controlled in such a way that it displaces proportionally to the applied force. Thus, applied force can be estimated from the displacement of the detection point and zero-compliance is accomplished at the point of force. The effectiveness of the proposed instrument in multi-dimensional force measurement is demonstrated by the experimental results of the horizontal and vertical direction force measurement with good linearity and almost zero interference.
\end{abstract}

Keywords : Zero compliance, Magnetic balance, Force measurement, Infinite stiffness, Series suspension, Electromagnetic force

\section{Introduction}

Force is one of the prime physical quantities in many industrial applications and scientific researches, especially in the development of new materials. Recently, there is a revolution in developing new materials such as paint, ink, cosmetic, concrete, etc. In such material development, the assembly of materials and molecules is essential. For precise assembling, interaction forces between molecules and surfaces are required to identify. In the world of miniaturization with high precision, the measurement of micro force becomes very important. There are several methods already established for force measurement (Stefanescu, 2011). MEMS sensors (Mei, et al., 2000; Fung, et al., 2002), piezoelectric actuators (Haddab, et al., 2000; Dao, et al., 2003; Woods, et al., 2001) and strain gauges (Haddab, et al., 2009; Peris, et al., 2004; Kemper, et al., 2004) are used in the research of small force measurement. Moreover, atomic force microscope cantilever is the most available tool for measuring small forces in diversified fields like material science to biological researches (Jalili and Laxminarayana, 2004; Surena, et al., 2013). Repulsive and attractive force can be estimated from the deflection of the cantilever. Most of the traditional methods measure the force from the displacement of the point of action in which the point of action is displaced from the source of force. In magnetic suspension balance, force is estimated from the control current without displacing the point of force (Boyden, et al., 1985; Sawada, et al., 1994). However, it has limitation in resolution because noise-signal ratio increases when force 
become small. To overcome this problem, zero-compliance force measurement using double series magnetic suspension is proposed (Mizuno, et al., 2011). In this method, high stiffness is accomplished at the point of force and high sensitivity is ensured in force measurement.

Based on the principle of zero-compliance force measurement, several devices have been developed using double series magnetic suspension. The first apparatus was built for vertical-direction force measurement (Mizuno, et al., 2014). In this system, an electromagnet was affixed at the top of the structure and two floators are levitated in series. The upper floator that is also known as detection point was connected with the base frame through leaf springs. In contrast, the bottom floator that is also denoted as point of force was suspended completely without contact. When force was applied to the point of force, zero-compliance was maintained at the point of force whereas the detection point was displaced in the direction of force. The applied force was estimated from the displacement of the detection point. Control current was also used to determine the force. The force measurement results demonstrated that the resolution of measuring force from control current was lower than that of force measurement using displacement. There are a few systems constructed for one-dimensional force measurement (Mizuno, et al., 2015; Hayashi, et al., 2016). The first work on multi-dimensional force measurement was developed for tri-axial force measurement using the similar principle of double series magnetic suspension (Mizuno, et al., 2016). As like the previous structure, the point of force was suspended completely without contact and the detection point was attached with leaf springs. Five electromagnets were used to control the motion in three directions. One electromagnet was used in the vertical direction and the other four were arranged in the horizontal directions. Permanent magnets were attached with a circular shaped detection point to maneuver the horizontal movements of the point of force. Force measurement results by this tri-axial force measurement apparatus attained zero compliance at the point of force and demonstrated linear displacement of the detection point in some extent. However, there were several limitations of this device because of the complete noncontact suspension of the point of force; the point of force was rotated even if in the zero compliance conditions, which influenced the measurement results. In addition, nonlinearity effect of the permanent magnet results in some inaccuracy in the measurement. Another three-component force measurement apparatus (Iida, et al., 2015) focused on measuring vertical direction axial force and rotation force along horizontal axes. To reduce the nonlinearity effect of the permanent magnet, voice coil motor was used in this device as linear actuator.

To accomplish stable zero compliance condition in multi-dimensional force measurement, this research focuses on developing a three-dimensional force ( $x-y-z$ axial force) measurement instrument and validates its performance by demonstrating the experimental results of vertical and horizontal directions force measurement. In this apparatus, both floators are suspended with leaf springs to restrain the rotation of point of force and to constrain its motions into translation. To reduce the nonlinearity effect of permanent magnet, voice coil motors are used in horizontal directions. A permanent magnet and an electromagnet are used only in the vertical direction.

\section{The Principle of force measurement}

\subsection{Zero-compliance mechanism}

Figure 1 presents the basic principle of force measurement using zero-compliance mechanism (Mizuno, et al, 2015). Two suspensions are connected in series, where the connection point $\mathrm{A}$ is the detection point and free end B is the point of force. The combined spring constant $\left(k_{c}\right)$ consists of individual stiffness of suspensions I $\left(k_{l}\right)$ and suspension II $\left(k_{2}\right)$ can be denoted as

$k_{c}=\frac{k_{1} k_{2}}{k_{1}+k_{2}}$

This equation demonstrates that if normal springs are connected, the resultant stiffness becomes lower than that the stiffness of suspension I and II. However, if the stiffness of the suspensions is set as shown by Fig. 1,
(b) $k_{1}>0$ and $k_{2}=-k_{1}<0$
or
(c) $k_{1}<0$ and $k_{2}=-k_{1}>0$

the combined stiffness becomes infinite. 


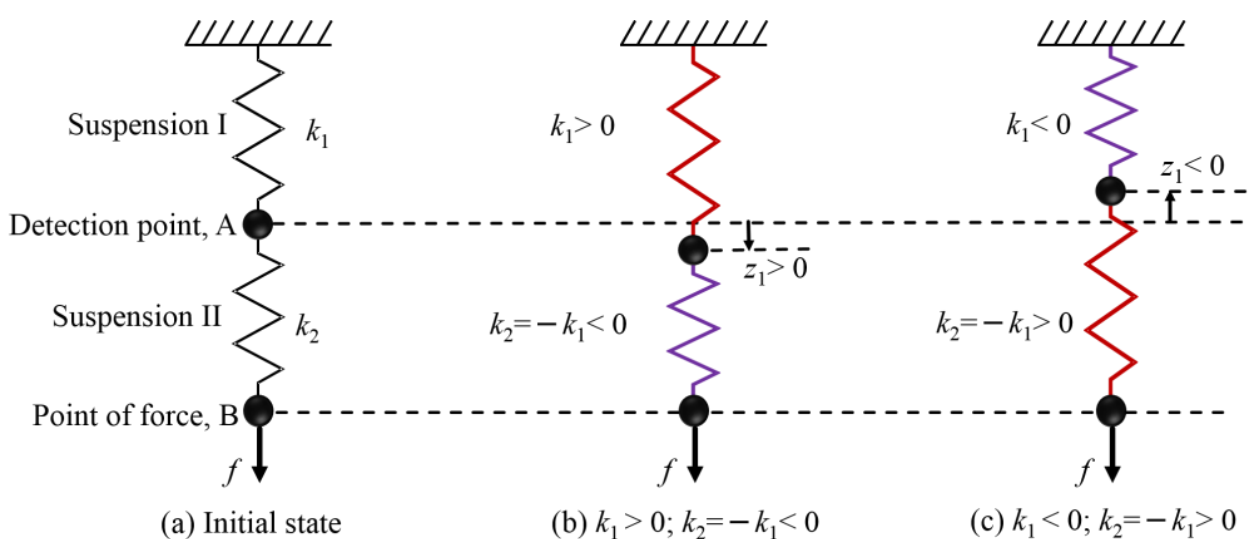

Fig. 1 Zero-compliance mechanism with series suspension. The free end, B is the point of force and the connection point A is the detection point. According to Fig. 1(b), when Suspension I has positive stiffness, the detection point displaces in the direction of the applied force. Figure 1(c) shows that when Suspension I has negative stiffness, the detection point displaces in the direction opposite to the applied force.
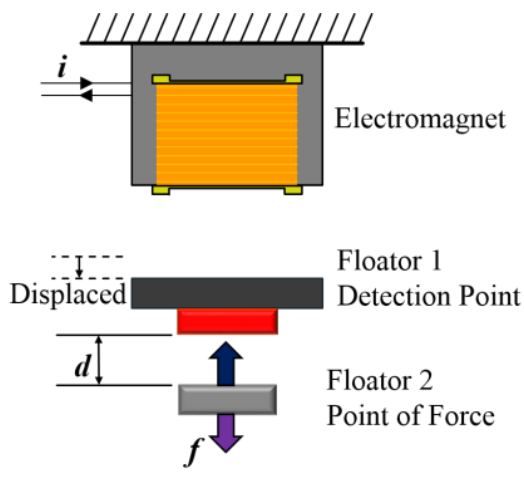

Fig. 2 Zero-compliance force measurement using double series magnetic suspension. An electromagnet directly controls Floator 1 and indirectly controls Floator 2 with the support of the permanent magnet (red block) installed at the bottom of the Floator 1. Applied force is measured from the displacement of the detection point.

$\left|k_{c}\right|=\left|\frac{k_{1}\left(-k_{1}\right)}{\left(k_{1}-k_{1}\right)}\right|=\infty$

That means the point of force will maintain its position even if force is applied, whereas the detection point will be displaced proportionally to the external force. As shown in Fig.1, if suspension I has positive stiffness, the detection point displacement will be in the direction of the applied force; in case of negative stiffness of suspension I, the detection point will move in the opposite direction of the external force.
(b) $z_{1}=\frac{f}{k_{1}}=-\frac{f}{k_{2}}>0$
or
(c) $z_{1}=\frac{f}{k_{1}}=-\frac{f}{k_{2}}<0$

Therefore, the applied force can be estimated from the displacement of the detection point.

\subsection{Double series magnetic suspension}

The basic principle of zero-compliance mechanism can be implemented in force measurement by using double series magnetic suspension. In this system, suspension of two floators is achieved by only one electromagnet as shown by Fig. 2. The control current of the electromagnet generates enough force to maneuver the position of the detection 
point. A permanent magnet (red block) is installed under the detection point. The attractive force of the permanent magnet controls the movement of the point of force. If the gap between the detection point and the point of force decreases, the attractive force of the permanent magnet increases, and the force decreases when the gap increases. Since the position of the detection point is directly controlled by the control current of the electromagnet, it also indirectly regulates the motion of the point of force. In this levitated condition, if any force is applied at the point of force, control current of the electromagnet changes to displace the detection point in the direction of applied force as shown by Fig. 1(b). Thus, the gap between the permanent magnet and the point of force changes to stabilize the motion of the point of force. The applied force can be estimated from the displacement of the detection point.

\subsection{Multi-dimensional force measurement}

Multi-dimensional force measurement is accomplished by elaborating the principle of double series magnetic suspension system. Vertical direction force measurement follows the similar configuration as shown by Fig. 2. In the lateral directions, zero-compliance force measurement is achieved by controlling the movement of the point of force and the displacements of the detection point in similar style of double series magnetic suspension system.

In the previously developed tri-axial force measurement device (Mizuno, et al., 2016) shown by Fig. 3, lateral motions were controlled by using four electromagnets. In contrast, four voice coil motors are arranged in the orientations as shown by Fig. 4 to control the movement of the detection point and the point of force in the horizontal

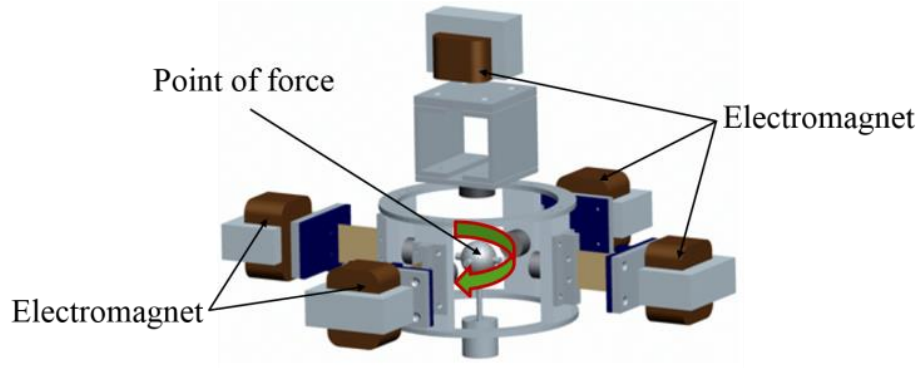

Fig. 3 Tri-axial force measurement device (Mizuno, et al., 2016). Five electromagnets are used to control the motions of the point of force in vertical and lateral directions. The point of force is completely suspended without any contact and it tends to rotate even in the zero-compliance conditions.

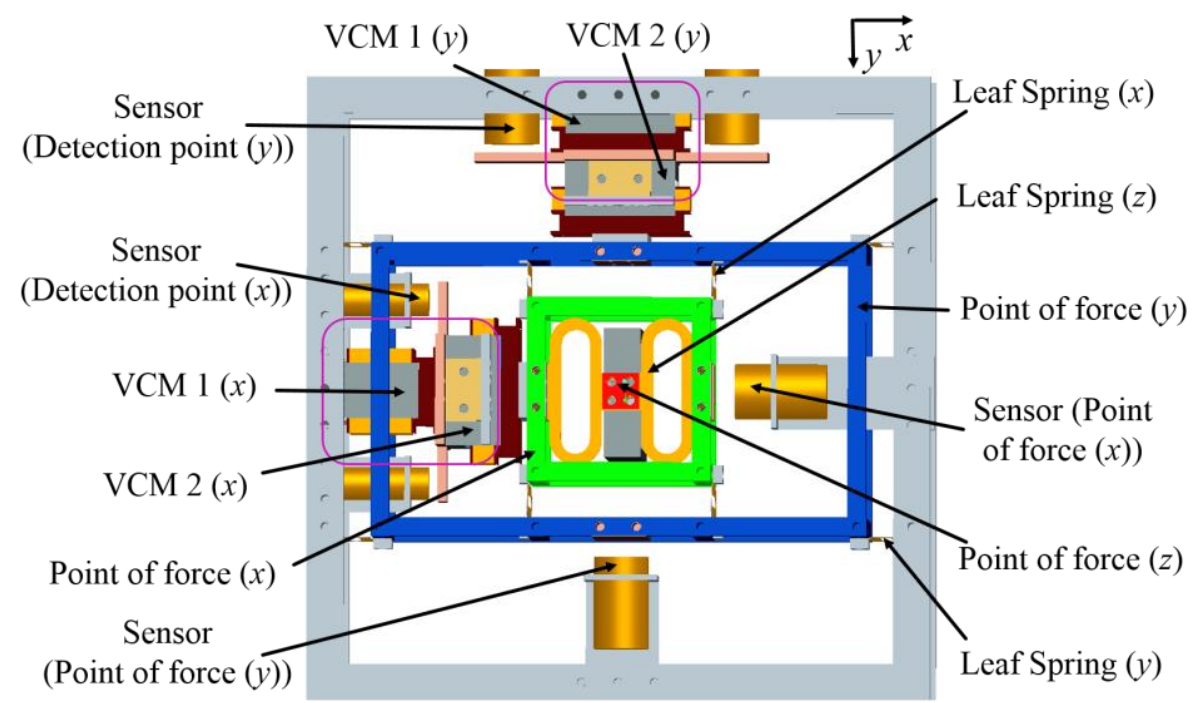

Fig. 4 Multi-dimensional force measurement arrangement. Two VCM assemblies (both consist of two VCMs) control the motion of the $x$ and $y$ directions. Red block, green frame and blue frames are the point of force in $x, y$ and $z$ directions, respectively. 
two- dimensions. Moreover, in the all three directions, both the point of force and the detection point are suspended from the base frame using leaf springs to restrict their motions in single dimension and to prevent the rotation of the point of force. On the contrary, the point of force was completely suspended without any contact in the previously developed device and it was rotating even if in the zero-compliance condition. As shown by Fig. 4, the center red block is the point of force in vertical $z$ direction, and green frame and blue frame are the point of force in the horizontal $x$ and $y$ directions respectively.

\section{Apparatus design and analysis}

\subsection{Design and fabrication}

To investigate multi-dimensional force measurement using zero compliance mechanism, a conventional-size apparatus was designed and fabricated (Nahiyan, et al., 2017). Figure 5 presents a photograph of the developed device. The schematic diagrams of the apparatus are shown by Fig. 6 to Fig. 9. Figure 6 demonstrates that the vertical direction force measurement is denoted as $z$ direction and the $x$ and $y$ axes are mentioned as the horizontal directions. As shown by Fig. 7, an electromagnet with 378 turns of $0.5 \mathrm{~mm}$-diameter coil is attached to the top frame to control the motion in the vertical direction. An iron plate suspended from the top frame by using leaf spring acts as the detection point ( $z$ ). A permanent magnet is adjoined under the extension part of the detection point to regulate the motion of the point of force in $z$ direction. An eddy-current gap sensor is attached to the bottom frame to measure the displacement of the point of force. From the top frame, two sensors are installed to estimate the movement of the detection point as shown by Fig. 8 . An extension frame and screw is attached under the point of force in the $z$ direction for adding weights on it to generate downward force.

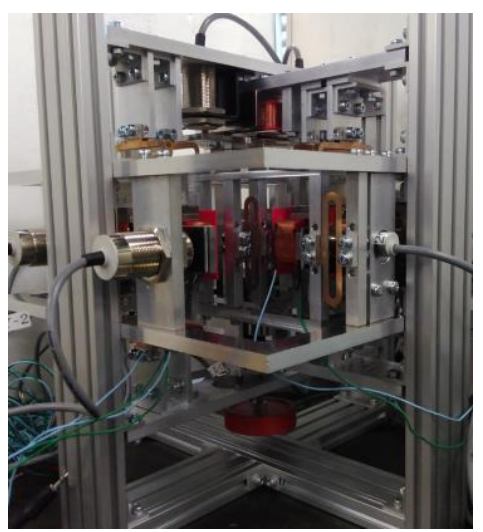

Fig. 5 Photograph of the multi-dimensional force measurement device designed and developed based on the zero-compliance mechanism.

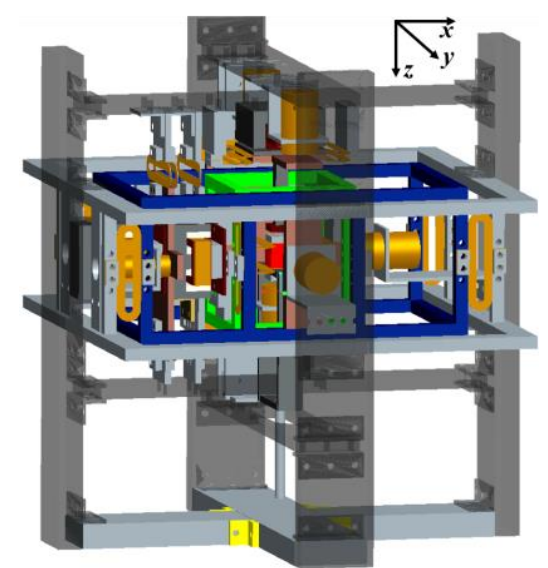

Fig. 6 Schematic diagram of the apparatus. The vertical direction is denoted as the $z$ axis and the lateral directions are represented as the $x$ and $y$ axes. 


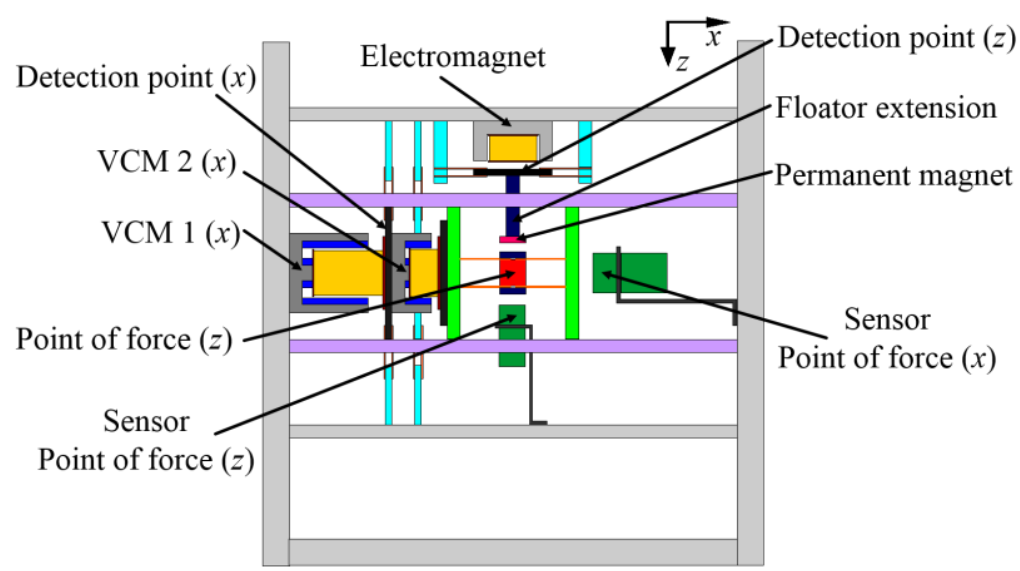

Fig. 7 Front view of the instrument. An electromagnet affixed at the top controls the displacement of the detection point $(z)$ and the permanent magnet attached at the bottom of the detection point $(z)$ regulates the deflection of the point of force $(z)$. In the $x$-direction mechanism, VCM $1(x)$ is fixed with the side frame and VCM $2(x)$ attached with the detection point $(x)$ and the bobbin of the VCM $1(x)$ VCM $2(x)$ and the detection point $(x)$ are suspended from top and bottom frame through leaf springs.

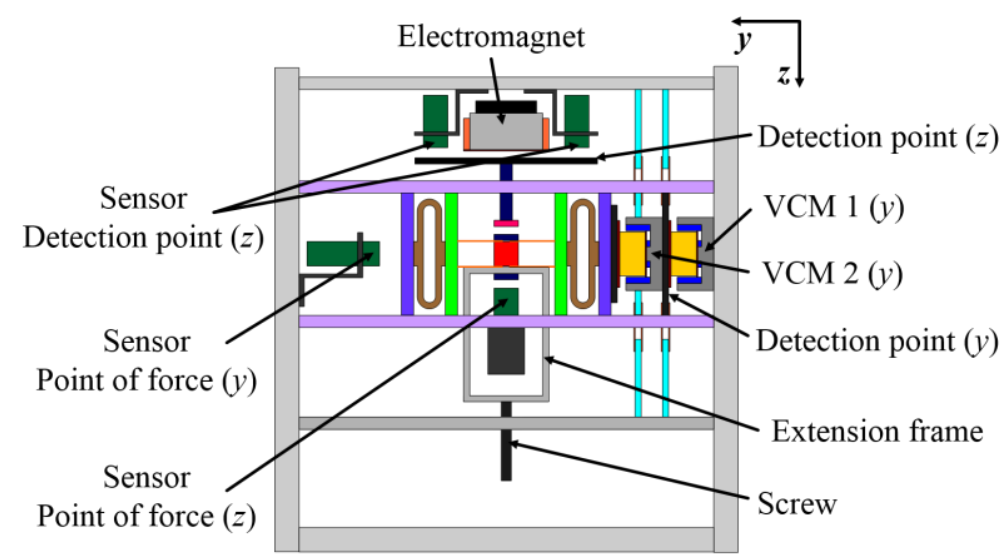

Fig. 8 Right-hand side view of the device. Two eddy current gap sensors, fixed in the top frame, measures the displacement of the detection point $(z)$ and another sensor attached at the bottom detects the movement of the point of force $(z)$. A screw is fixed to the extension frame of the point of force $(z)$, to apply external force.

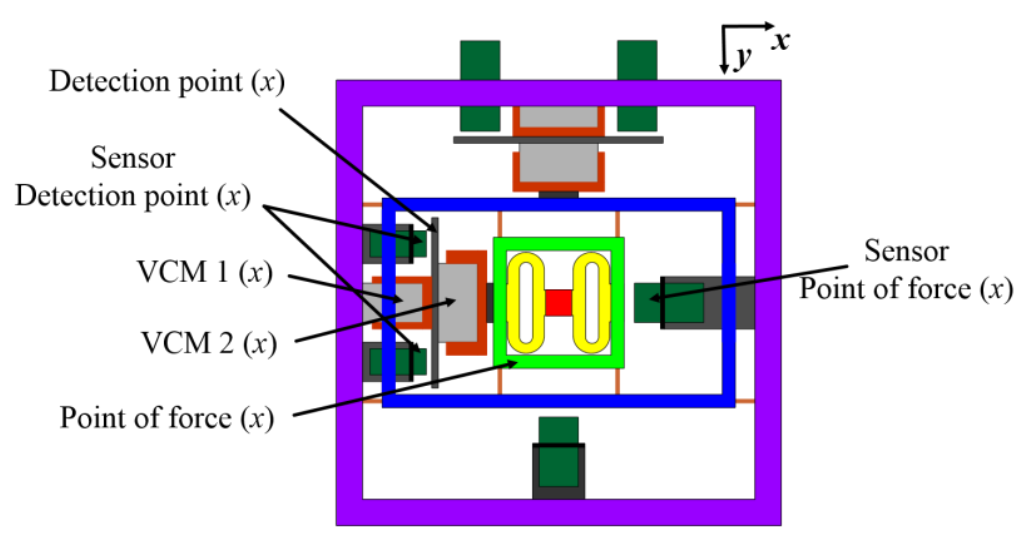

Fig. 9 Bottom view. Both in the $x$ and $y$ directions, two sensors detect the displacement of the detection point and another sensor measure the deflection of the point of force. The bobbin of VCM $1(x)$ is attached to the detection point and the bobbin of VCM $2(x)$ is connected to the green frame. 
Voice coil motors are used to control the motion in the horizontal $x$ and $y$ directions. In each axis, two voice coil motors are used to control the movement of the detection point and the point of force as shown by Fig. 9. As shown in Fig. 7 and Fig. 9, in the $x$ direction, core of VCM $1(x)$ is fixed with the outer base frame. The bobbin of the VCM $1(x)$, detection plate and the core of VCM $2(x)$ are connected to the upper and lower base frame through leaf springs. The bobbin of VCM $2(x)$ are fixed with the point of force $(x)$. The bobbin of VCM $2(x)$ is large enough to allow the green frame to move in $y$ direction along with the blue frame. In the force measurement operation, VCM $1(x)$ regulates the displacement of the detection point and VCM $2(x)$ controls the motion of the point of force. The point of force is connected to the fixed outer frame through leaf springs. Similar to the vertical direction, two eddy-current gap sensors measure the displacement of the detection point and another sensor estimates the movement of the point of force. Principle of $y$ direction operation is similar to the $x$-direction mechanism.

\subsection{Mathematical model and controllers}

In the coordinate system of the device, $z$ axis is mentioned for the vertical direction and the horizontal plane is denoted by the $x$ axis and $y$ axis as shown by Fig. 6. A simple schematic diagram of the $z$ direction force measurement can be shown as Fig. 10.

The equations of motion in the vertical direction are given by

$$
\begin{aligned}
& m_{1}^{(z)} \ddot{z}_{1}(t)=-\left(h_{1}^{(z)}-k_{s}^{(z)}\right) z_{1}+k_{m}^{(z)}\left(z_{1}-z_{2}\right)-k_{i}^{(z)} i^{(z)} \\
& m_{2}^{(z)} \ddot{z}_{2}(t)=-h_{2}^{(z)} z_{2}-k_{m}^{(z)}\left(z_{1}-z_{2}\right)+f^{(z)}(t)
\end{aligned}
$$

where $m_{1}^{(z)}$ and $m_{2}^{(z)}$ : mass of the detection point $(z)$ and mass of the point of force $(z) ; h_{1}^{(z)}$ and $h_{2}^{(z)}:$ stiffness of leaf springs attached with the detection point $(z)$ and stiffness of leaf springs connected with the point of force $(z)$; $z_{1}$ and $z_{2}$ : displacement of the detection point $(z)$ and displacement of the point of force $(z), k_{i}^{(z)}$ and $k_{s}^{(z)}$ : current force factor and gap-force factor of electromagnet; $k_{m}^{(z)}$ : gap force factor of permanent magnet, $f^{(z)}$ : external force acting on the point of force.

In zero-compliance force measurement using double series magnetic suspension, force is measured from the displacement of the detection point and the point of force maintains its position. To maintain the position of the point of force, PID control is applied to the point of force, whereas PD control to the detection point makes the total system stable. By combining these PD and PID control, the control current for the $z$ direction motion is given by

$$
I^{(z)}(s)=\left(p_{d}^{(z)}+s p_{v}^{(z)}\right) Z_{1}(s)-\left(q_{d}^{(z)}+s q_{v}^{(z)}+\frac{q_{I}^{(z)}}{s}\right) Z_{2}(s)
$$
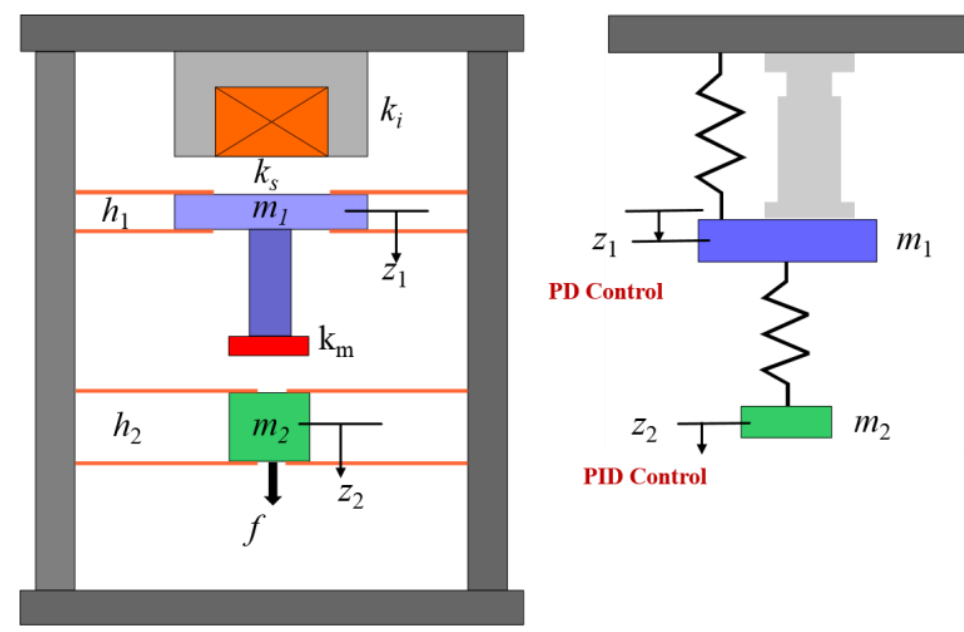

Fig. 10 Schematic diagram of the $z$ direction force measurement. Both the detection point of mass $m_{1}$ and the point of force of mass $m_{2}$ are suspended from the base frame through leaf springs of stiffness $h_{1}$ and $h_{2}$. 


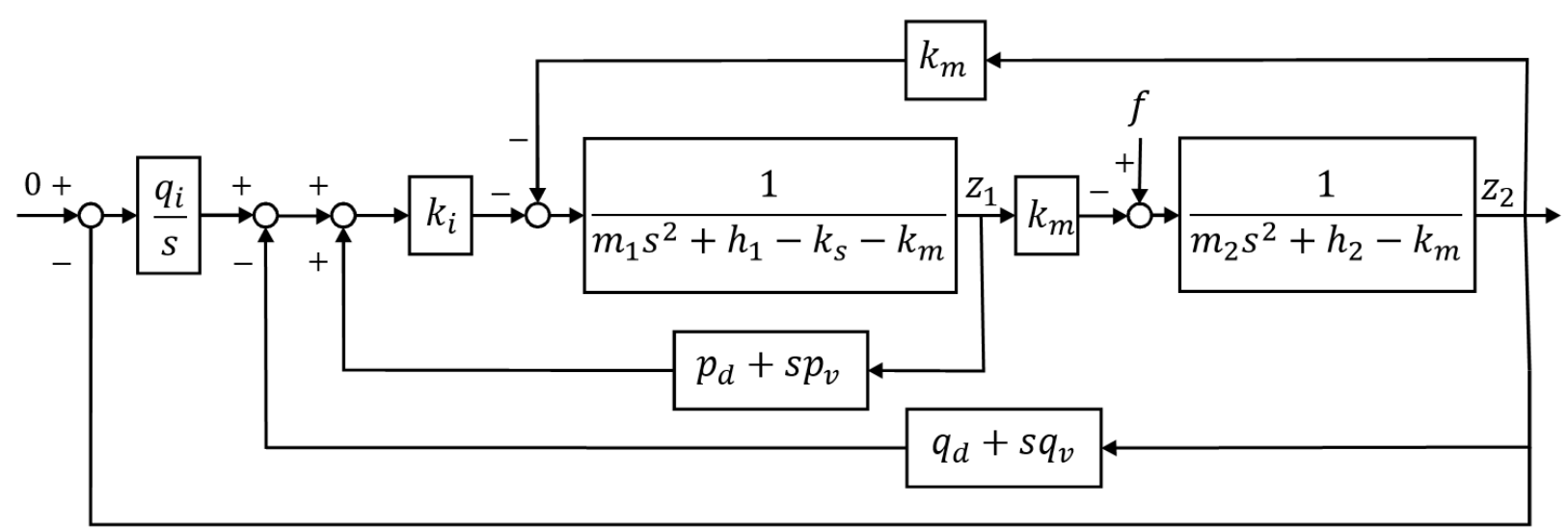

Fig. 11 Control system block diagram for the $z$-direction movement. PD control is applied to the detection point and PID control is applied to the point of force.

where $p_{d}^{(z)}$ and $p_{v}^{(z)}$ are the proportional gain and derivative gain of PD controller of the detection point $(z)$, and $q_{d}^{(z)}$, $q_{v}^{(z)}$ and $q_{I}^{(z)}$ are the proportional gain, derivative gain, and integral gain of the point of force $(z)$, respectively. Figure 11 shows the control system block diagram to control the $z$ direction movement.

Table 1 and Table 2 present the values of the physical and the control paraments of the $z$-direction force measurement, respectively.

Table 1 Values of the physical parameters in $z$ direction

Table 2 Values of the control parameters in $z$ direction

\begin{tabular}{c|c|c}
\hline Physical parameter & Numerical value & Unit \\
\hline$m_{1}$ & 0.489 & $\mathrm{~kg}$ \\
$m_{2}$ & 0.241 & $\mathrm{~kg}$ \\
$k_{i}$ & 7.5 & $\mathrm{~N} / \mathrm{A}$ \\
$k_{s 1}\left(=h_{1}-k_{s}\right)$ & 8009 & $\mathrm{mN} / \mathrm{mm}$ \\
$k_{m}$ & 1023 & $\mathrm{mN} / \mathrm{mm}$ \\
$h_{2}$ & 8284 & $\mathrm{mN} / \mathrm{mm}$ \\
\hline
\end{tabular}

\begin{tabular}{c|c|c}
\hline Physical parameter & Numerical value & Unit \\
\hline$p_{\mathrm{d}}$ & 100 & $\mathrm{~A} / \mathrm{m}$ \\
$p_{\mathrm{v}}$ & 0.1 & $\mathrm{As} / \mathrm{m}$ \\
$q_{\mathrm{d}}$ & 100 & $\mathrm{~A} / \mathrm{m}$ \\
$q_{\mathrm{v}}$ & 0.1 & $\mathrm{As} / \mathrm{m}$ \\
$q_{\mathrm{i}}$ & 500 & $\mathrm{~A} / \mathrm{ms}$ \\
Bias Current & 0.6 & $\mathrm{~A}$ \\
\hline
\end{tabular}

Figure 12 presents the schematic diagram of the $x$ direction force measurement. The equations of motion in $x$ direction can be represented as

$m_{1}^{(x)} \ddot{x}_{1}(t)=-h_{1}^{(x)} x_{1}+k_{i 1}^{(x)} \dot{i}_{1}^{(x)}-k_{i 2}^{(x)} \dot{i}_{2}^{(x)}$

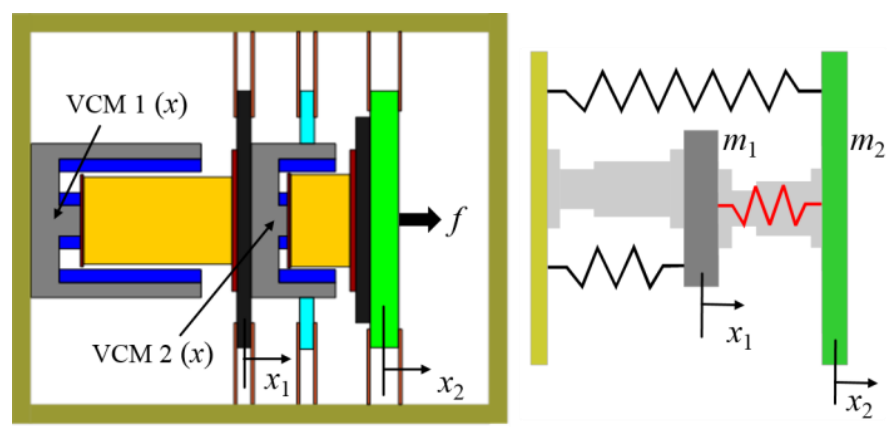

Fig. 12 Schematic diagram of the $x$-direction force measurement. The core of VCM $1(x)$ is fixed to the base frame. The bobbin of VCM $1(x)$ and the core of VCM $2(x)$ are attached to the detection point and suspended from the top and the bottom frame through leaf springs. The bobbin of the VCM $2(x)$ is connected with the green frame. 


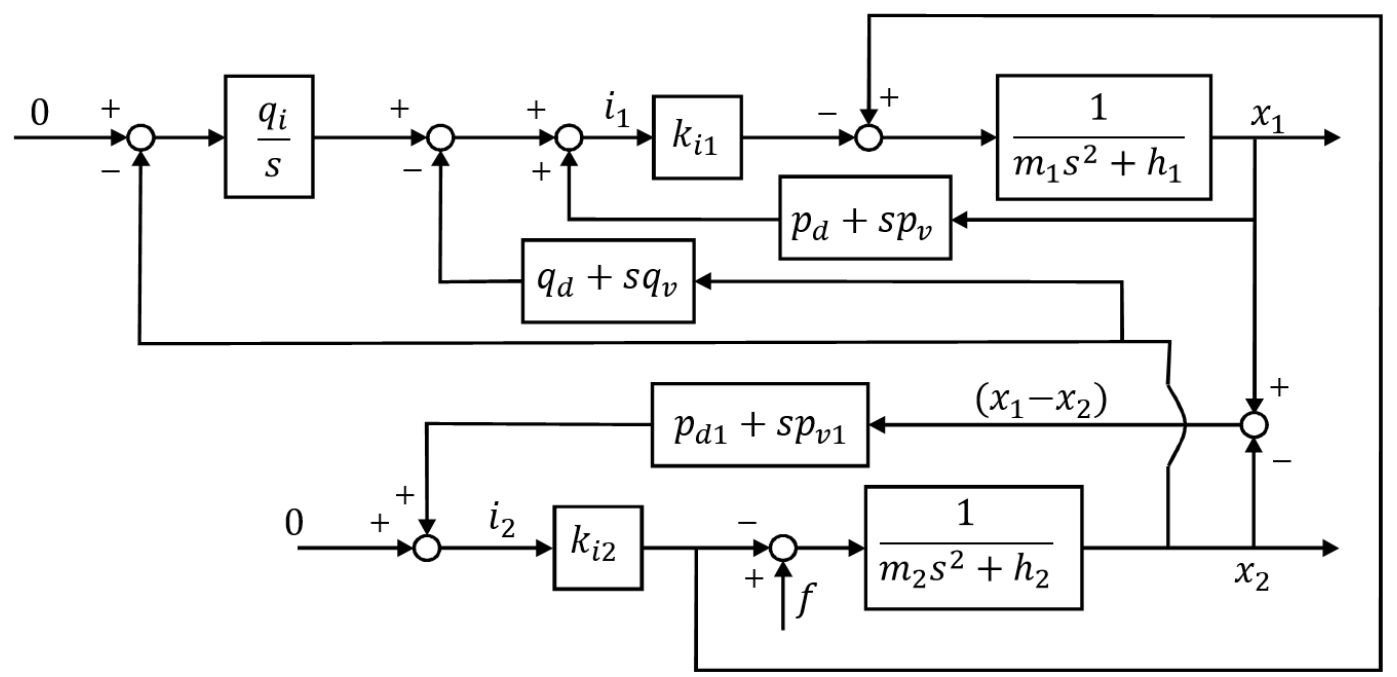

Fig. 13 Block diagram of control system for the $x$ direction motion. PD control is applied to the detection point and PID control is applied to the point of force. Additional PD control is applied in between the detection point and the point of force.

$m_{2}^{(x)} \ddot{x_{2}}(t)=-h_{2}^{(x)} x_{2}+k_{i 2}^{(x)} i_{2}^{(x)}+f^{(x)}(t)$

where $m_{1}^{(x)}$ and $m_{2}^{(x)}$ are the mass of the detection point $(x)$ and the point of force $(x) ; h_{1}^{(x)}$ and $h_{2}^{(x)}$ are the stiffness of leaf springs attached with the detection point $(x)$ and leaf springs connected with the point of force $(x) ; x_{1}$ and $x_{2}$ are the displacement of the detection point $(x)$ and the point of force $(x) ; k_{i 1}^{(x)}$ and $k_{i 2}^{(x)}$ are the current force factor of voice coil motor 1 and $2 ; i_{1}^{(x)}$ and $i_{2}^{(x)}$ are the control current of VCM 1 and 2; and $f^{(x)}$ is the external force acting on the point of force $(x)$.

In contrast to the $z$ direction, there is no permanent magnet in the $x$ direction to generate negative stiffness. Thus, an additional PD control is imposed in between the detection point and the point of force to act as virtual spring with positive stiffness. According to Fig. 1(c), the detection point displaces in the direction opposite to the applied force.

The control current for zero-compliance force measurement in the $x$ direction can be given by

$I_{1}^{(x)}(s)=\left(p_{d}^{(x)}+s p_{v}^{(x)}\right) X_{1}(s)-\left(q_{d}^{(x)}+s q_{v}^{(x)}+\frac{q_{I}^{(x)}}{s}\right) X_{2}(s)$

$I_{2}^{(x)}(s)=\left(p_{d 1}^{(x)}+s p_{v 1}^{(x)}\right)\left\{X_{1}(s)-X_{2}(s)\right\}$

where $p_{d}^{(x)}$ and $p_{v}^{(x)}$ are the proportional gain and derivative gain of PD controller of the detection point $(x)$, and $q_{d}^{(x)}$, $q_{v}^{(x)}$ and $q_{I}^{(x)}$ are the proportional gain, derivative gain, and integral gain of the point of force $(x)$, respectively. $p_{d 1}^{(x)}$ and $p_{v 1}^{(x)}$ are the proportional gain and derivative gain of the additional PD controller to generate the virtual

Table 3 Values of the physical parameter in $x$ direction

\begin{tabular}{c|c|c}
\hline Physical parameter & Numerical value & Unit \\
\hline$m_{1}$ & 1.001 & $\mathrm{~kg}$ \\
$m_{2}$ & 0.776 & $\mathrm{~kg}$ \\
$k_{i l}$ & 8.5 & $\mathrm{~N} / \mathrm{A}$ \\
$k_{i 2}$ & 9.97 & $\mathrm{~N} / \mathrm{A}$ \\
$h_{1}$ & 4702 & $\mathrm{mN} / \mathrm{mm}$ \\
$h_{2}$ & 5877 & $\mathrm{mN} / \mathrm{mm}$ \\
\hline
\end{tabular}

Table 4 Values of the control parameter in $x$ direction

\begin{tabular}{c|c|c}
\hline Physical parameter & Numerical value & Unit \\
\hline$p_{\mathrm{d}}$ & 100 & $\mathrm{~A} / \mathrm{m}$ \\
$p_{\mathrm{v}}$ & 0.1 & $\mathrm{As} / \mathrm{m}$ \\
$q_{\mathrm{d}}$ & 100 & $\mathrm{~A} / \mathrm{m}$ \\
$q_{\mathrm{v}}$ & 0.1 & $\mathrm{As} / \mathrm{m}$ \\
$q_{\mathrm{i}}$ & 500 & $\mathrm{~A} / \mathrm{ms}$ \\
$p_{\mathrm{d} 1}$ & 100 & $\mathrm{~A} / \mathrm{m}$ \\
$p_{\mathrm{v} 1}$ & 1 & $\mathrm{As} / \mathrm{m}$ \\
\hline
\end{tabular}


spring imposed in between the detection point and the point of force. The block diagram of control system for the $x$ axis motion is shown by Fig. 13.

Table 3 and Table 4 present the values of the physical and the control parameters of the $x$-direction force measurement, respectively.

\subsection{Analysis using transfer functions}

By using the Laplace transformation of the equations of motion for the $z$ axis movement, transfer function can be derived as

$$
\begin{aligned}
& Z_{1}(s)=\frac{A_{1}^{(z)}(s)}{D^{(z)}(s)} F^{(z)}(s) \\
& Z_{2}(s)=\frac{A_{2}^{(z)}(s)}{D^{(z)}(s)} F^{(z)}(s)
\end{aligned}
$$

where

$$
\begin{aligned}
A_{1}^{(z)}(s)= & k_{i}^{(z)} q_{v}^{(z)} s^{2}+\left(k_{i}^{(z)} q_{d}^{(z)}-k_{m}^{(z)}\right) s+k_{i}^{(z)} q_{I}^{(z)} \\
A_{2}^{(z)}(s)= & m_{1}^{(z)} s^{3}+k_{i}^{(z)} p_{v}^{(z)} s^{2}+\left(k_{i}^{(z)} p_{d}^{(z)}+k_{s 1}^{(z)}-k_{m}^{(z)}\right) s \\
D^{(z)}(s)= & m_{1}^{(z)} m_{2}^{(z)} s^{5}+m_{2}^{(z)} k_{i}^{(z)} p_{v}^{(z)} s^{4}+\left\{m_{1}^{(z)}\left(h_{2}^{(z)}-k_{m}^{(z)}\right)+m_{2}^{(z)}\left(k_{s 1}^{(z)}-k_{m}^{(z)}+k_{i}^{(z)} p_{d}^{(z)}\right)\right\} s^{3} \\
& +\left\{k_{i}^{(z)} p_{v}^{(z)}\left(\left(k_{m}^{(z)}-h_{2}^{(z)}\right)+k_{m}^{(z)} k_{i}^{(z)} q_{v}^{(z)}\right\} s^{2}+\right. \\
& \left\{h_{2}^{(z)}\left(k_{s 1}^{(z)}+k_{i}^{(z)} p_{d}^{(z)}-k_{m}^{(z)}\right)+k_{m}^{(z)}\left(k_{i}^{(z)} q_{d}^{(z)}-k_{s 1}^{(z)}-k_{i}^{(z)} p_{d}^{(z)}\right)\right\} s+k_{m}^{(z)} k_{i}^{(z)} q_{I}^{(z)} \\
k_{s 1}^{(z)}= & h_{1}^{(z)}-k_{s}^{(z)}
\end{aligned}
$$

From Eq. (16), signs of the control parameter $p_{d}^{(z)}, p_{v}^{(z)}, q_{d}^{(z)}, q_{v}^{(z)}$ and $q_{I}^{(z)}$ can be selected by using pole placement method.

When the applied force acting on the point of force is a step function,

$$
F^{(z)}=\frac{F_{0}^{(z)}}{s}
$$

$F_{0}^{(z)}$ is constant. The displacement in the steady-state condition can be represented as

$$
\begin{aligned}
& z_{1}(\infty)=\lim _{t \rightarrow \infty} z_{1}(t)=\lim _{s \rightarrow 0} s Z_{1}=\frac{F_{0}^{(z)}}{k_{m}^{(z)}} \\
& z_{2}(\infty)=\lim _{t \rightarrow \infty} z_{2}(t)=\lim _{s \rightarrow 0} s Z_{2}=0
\end{aligned}
$$

It is shown by Eq. (19) that there is no displacement at the point of force, which means that it is suspended by spring with infinite stiffness. Even though force is applied at the point of force, it maintains its position. Moreover, Eq. (18) presents that the detection point moved in the direction of the applied force. Thus, the total suspension in the $z$ direction follows the principle of zero-compliance force measurement using double series magnetic suspension.

The transfer function in the $x$ direction are given by

$$
X_{1}(s)=\frac{B_{1}^{(x)}(s)}{D^{(x)}(s)} F^{(x)}(s)
$$




$$
X_{2}(s)=\frac{B_{2}^{(x)}(s)}{D^{(x)}(s)} F^{(x)}(s)
$$

where

$$
\begin{aligned}
B_{1}^{(x)}(s)= & \left(k_{i 2}^{(x)} p_{v 1}^{(x)}-k_{i 1}^{(x)} q_{v}^{(x)}\right) s^{2}+\left(k_{i 2}^{(x)} p_{d 1}^{(x)}-k_{i 1}^{(x)} q_{d}^{(x)}\right) s-k_{i 1}^{(x)} q_{I}^{(x)} \\
B_{2}^{(x)}(s)= & m_{1} s^{3}+\left(k_{i 2}^{(x)} p_{v 1}^{(x)}-k_{i 1}^{(x)} p_{v}^{(x)}\right) s^{2}+\left(h_{1}^{(x)}-k_{i 1}^{(x)} p_{d}^{(x)}+k_{i 2}^{(x)} p_{d 1}^{(x)}\right) s \\
D^{(x)}(s)= & m_{1}^{(x)} m_{2}^{(x)} s^{5}+\left\{m_{2}^{(x)}\left(k_{i 2}^{(x)} p_{v 1}^{(x)}-k_{i 1}^{(x)} p_{v}^{(x)}\right)+m_{1}^{(x)} k_{i 2}^{(x)} p_{v 1}^{(x)}\right\} s^{4}+ \\
& \left\{m_{1}^{(x)}\left(h_{2}^{(x)}+k_{i 2}^{(x)} p_{d 1}^{(x)}\right)+m_{2}^{(x)}\left(h_{1}^{(x)}-k_{i 1}^{(x)} p_{d}^{(x)}+k_{i 2}^{(x)} p_{d 1}^{(x)}\right)+k_{i 1}^{(x)} k_{i 2}^{(x)} p_{v 1}^{(x)}\left(q_{v}^{(x)}-p_{v}^{(x)}\right) s^{3}+\right. \\
& \left\{k_{i 2}^{(x)} p_{v 1}^{(x)}\left(k_{i 1}^{(x)} q_{d}^{(x)}+h_{1}^{(x)}+h_{2}^{(x)}-k_{i 1}^{(x)} p_{d}^{(x)}\right)-k_{i 1}^{(x)} p_{v}^{(x)}\left(h_{2}^{(x)}+k_{i 2}^{(x)} p_{d 1}^{(x)}\right)+k_{i 1}^{(x)} k_{i 2}^{(x)} p_{d 1}^{(x)} q_{v}^{(x)}\right\} s^{2}+ \\
& \left\{\left(h_{2}^{(x)}+k_{i 2}^{(x)} p_{d 1}^{(x)}\right)\left(h_{1}^{(x)}-k_{i 1}^{(x)} p_{d}^{(x)}\right)+k_{i 2}^{(x)} p_{d 1}^{(x)}\left(k_{i 1}^{(x)} q_{d}^{(x)}+h_{2}^{(x)}\right)+k_{i 1}^{(x)} k_{i 2}^{(x)} p_{v 1}^{(x)} q_{I}^{(x)}\right\} s+k_{i 1}^{(x)} k_{i 2}^{(x)} p_{d 1}^{(x)} q_{I}^{(x)}
\end{aligned}
$$

The steady-state displacement in the $x$ direction can be represented as

$$
\begin{aligned}
& x_{1}(\infty)=\lim _{t \rightarrow \infty} x_{1}(t)=\lim _{s \rightarrow 0} s X_{1}(s)=-\frac{F_{0}^{(z)}}{k_{i 2}^{(x)} p_{d 1}^{(x)}} \\
& x_{2}(\infty)=\lim _{t \rightarrow \infty} x_{2}(t)=\lim _{s \rightarrow 0} s X_{2}(s)=0
\end{aligned}
$$

Equation (26) confirms that, zero compliance can be achieved at the point of force in the $x$ direction as well as in the $z$ axis. In contrast to Eq. (18), a minus sign appears in the right-hand side of Eq. (25). Usually the stiffness is set to be positive by PD control to stabilize the system, which indicates that the detection point moves in the direction opposite to the applied force as shown by Fig. 1(c).

\section{Experimental results and discussion}

The Force measurement experiments are carried out by applying static force. The instrument is tilted around the $y$ axis by $45^{\circ}$ to measure force in the vertical and horizontal directions. Weights are added at the point of force in the $z$

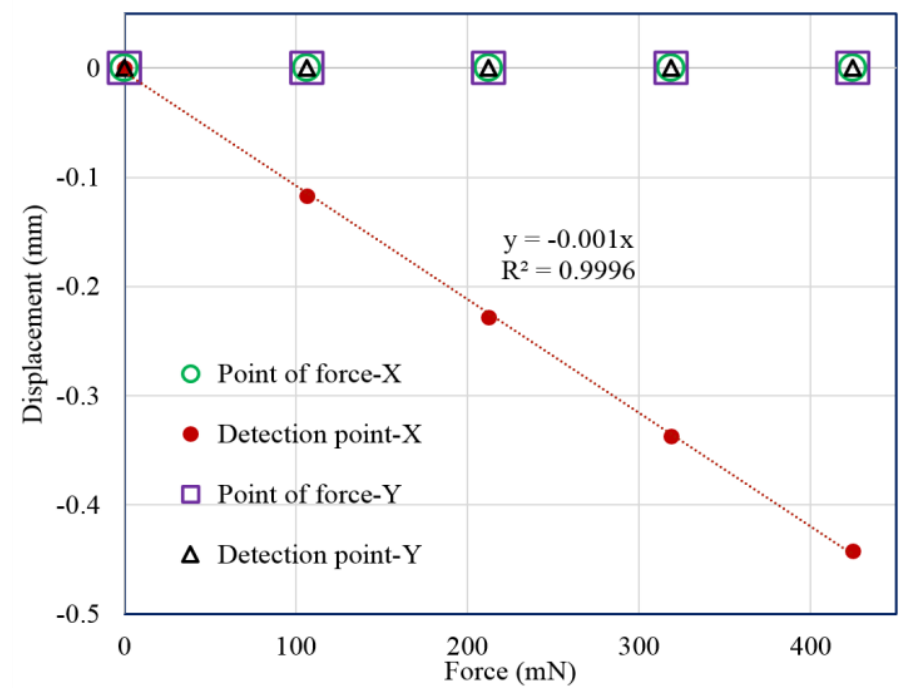

Fig. 14 Displacement of the detection point $(x)$, point of force $(x)$, detection point $(y)$ and point of force $(y)$ with the addition of force in the $x$ direction. Blue dot, red circle, green triangle and black rectangle present the detection point $(x)$, point of force $(x)$, detection point $(y)$ and point of force $(y)$, respectively. The detection point $(x)$ displaces in the direction opposite to the applied force. 
direction and the components of the applied force are taken into consideration both in the $x$ and $z$ axes. $y$ direction force measurement can also be accomplished in similar fashion by tilting the device in Y-Z plane.

Figure 14 presents the displacement of the point of force and the detection point in the $x$ and $y$ directions and Fig. 15 shows the change of control current corresponding to the addition of force in the $x$ axis. It is shown by Fig. 14 that with the addition of force, the detection point $(x)$ moves in the opposite direction of force whereas the point of force $(x)$ keeps its position. Force is increased by adding weights one by one. The experimental results of adding 10.8-g weight one by one are displayed in Fig. 14 and Fig. 15. Additionally, Fig. 14 confirms that there is no interference on the point of force $(y)$ and the detection point $(y)$ with the addition of force in the $x$ direction. Moreover, the experimental result satisfies the theoretical analysis of Eq. (25), that if the applied force is increased, the detection point displacement increases in the direction opposite to the applied force.

From the same experiment, graphs are plotted with the data of the $z$ direction force measurement. Figure 16 shows the movement of the detection point and the displacement of the point of force in the $z$ and $y$ directions. With the addition of force, the point of force $(z)$ maintains its position as in the $x$ axis and the detection point $(z)$ moves downward in proportion of the force. The relationship between the control current and applied force in the $z$ direction is

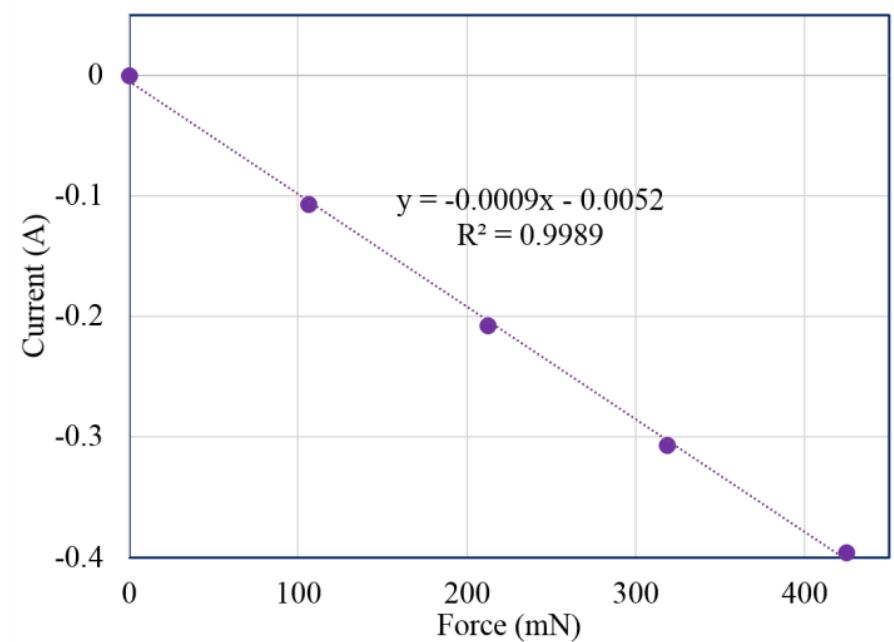

Fig. 15 Relationship between the control current of VCM $1(x)$ and the force applied in the $x$ direction.

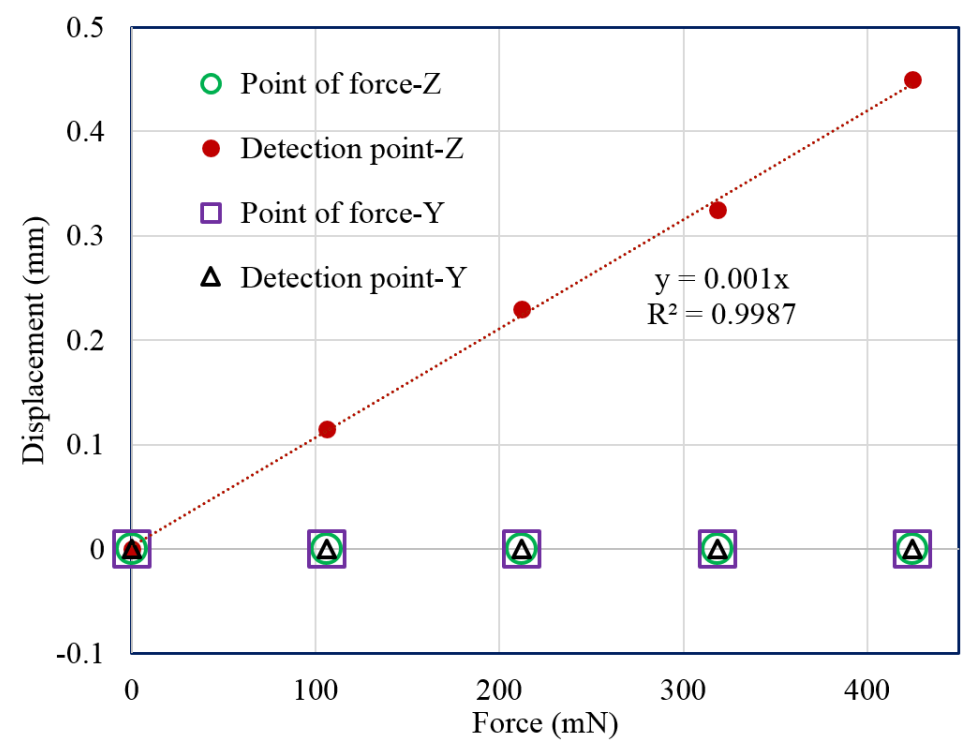

Fig. 16 Displacement of the detection point $(z)$, point of force $(z)$, detection point $(y)$ and point of force $(y)$ with the addition of force in the $z$ direction. Blue dot, red dot, black rectangle and green triangle present the detection point $(z)$, point of force $(z)$, detection point $(y)$ and point of force $(y)$, respectively. The detection point $(z)$ displaces in the same direction of the applied force. 


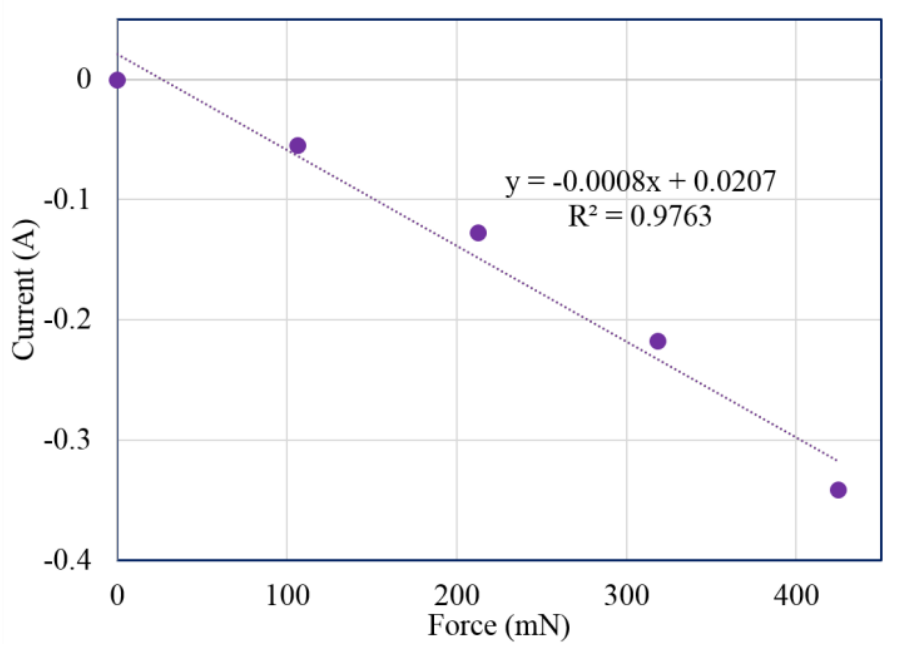

Fig. 17 Relationship between the control current of the electromagnet and the force applied in the $z$ direction.

shown by Fig. 17. Similar to the $x$ axis force measurement, Fig.16 represent that there is no interference in the $y$ direction while force is applied in the $z$ direction. The experimental result of $z$-direction also satisfies the theoretical analysis of Eq. (18), that is if the applied force is increased, the detection point displacement increases in the same direction of the applied force.

The results of the experiment confirm that stable zero-compliance is achieved at the point of force both in the $z$ and $x$ directions. Additionally, good linearity is shown in the displacement of the detection point. In comparison to the movement of the detection point, there is some nonlinearity in the change of control current as shown in Fig. 15 and Fig. 17.

\section{Conclusion}

Multi-dimensional force measurement using zero compliance mechanism was validated from the experimental results. To achieve stable zero-compliance at the point of force, a novel multi-dimensional force measurement instrument was designed and fabricated. The control system was designed and implemented for force measurement both in the vertical and horizontal direction. The device was tilted around the $y$ axis by $45^{\circ}$ and weights were added to the point of force $(\mathrm{z})$ to investigate the performance of the apparatus under the application of static force. Multi-dimensional force measurement experiment was carried out by gradually increasing and decreasing weights. PD control of the detection point and PID control of the point of force was applied for force measurement in $z$ axis. Additionally, another PD control was employed in between the detection point and the point of force in $x$ direction to act as a virtual spring. The results of force measurement demonstrated that both in the $x$ and $z$ directions, stable zero-compliance was achieved at the point of force and the detection point displaced with good linearity. Thus, from the displacement of the detection point, force was estimated. Additionally, by selecting softer leaf springs or displacement sensor with smaller resolution, small amount of force can be estimated with good accuracy.

\section{Acknowledgement}

This work was supported by JSPS KAKENHI (Grant Number: JP17H03188).

\section{References}

Boyden, R. P., Britcher, C. P. and Tcheng, P., Status of Wind Tunnel Magnetic Suspension Research. SAE Technical Paper Series 851898 (1985), pp. 1-9.

Dao, D. V., Toriyama, T., Wells, J. and Sugiyama, S., Silicon Piezoresistive Six Degree of Freedom Force-Moment Microsensor. Sens. Mater., Vol.15, No.3 (2003), pp.113-135. 
Fung, C. K. M., Elhajj, I. H., Li, W. J. and Xi, N., A 2-D PVDF Force Sensing System for Micromanipulation and Micro-Assembly. Proceedings of the IEEE International Conference on Robotics and Automation (2002), pp.1489-1494.

Haddab, Y., Chaillet, N. and Bourjault, A., A Microgripper using Smart Piezoelectric Actuators. Proceedings of the IEEE/RSJ International Conference on Intelligent Robots and Systems (2000), pp.659-664.

Haddab, Y., Chen, Q. and Lutz, P., Improvement of Strain Gauges Micro-Forces Measurement using Kalman Optimal Filtering. Mechatronics, Vol.19, No.4 (2009), pp.457-462.

Hayashi, Y., Takasaki, M., Ishino, Y., Yamaguchi, D., Hara, M. and Mizuno, T., Development of Force Measurement System using Zero-Compliance Mechanism with a Cantilever ( $2^{\text {nd }}$ Report: Fabrication of Measurement Mechanism). Proceedings of 25th MAGDA Conference (2016), pp.451-453 (in Japanese).

Iida, K., Mizuno, T., Takasaki, M., Ishino, Y., Yamaguchi, D. and Hara, M., Development of 3-Component Force Measurement Apparatus Using Zero-Compliance Mechanism (1sr Report: Basic Concepts and Fabrication of Measurement Device). Proceedings of Dynamics and Design Conference (2015) (in Japanese).

Jalili, N. and Laxminarayana, K., A Review of Atomic Force Microscopy Imaging Systems: Application to Molecular Metrology and Biological Sciences. Mechatronics, Vol.14 (2004), pp.907-945.

Kemper, M., Development of A Tactile Low-Cost Microgripper with Integrated Force Sensor. Proceedings of the IEEE International Conference on Control Applications, Taipei, Taiwan (2004), pp.1461-1466.

Mei, T., Li, W. J., Ge, Y., Chen, Y., Ni, L. and Chan, M. H., An Integrated MEMS Three-Dimensional Tactile Sensor with Large Force Range. Sensors and Actuators A, Vol.80 (2000), pp.155-162.

Mizuno, T., Sekine, D., Ishino, Y. and Takasaki, M., Noncontact Microforce Measurement using Series Magnetic Suspension ( $2^{\text {nd }}$ Report: Fabrication of an Experimental Apparatus). Proceedings of Dynamics and Design Conference (2011), (in Japanese).

Mizuno, T., Sekine, D., Takasaki, M. and Ishino, Y., Force Measurement using Double Series Magnetic Suspension. Transaction of the Japan Society of Mechanical Engineers, Vol.80, No.814 (2014), (in Japanese).

Mizuno, T., Hayashi, Y., Ishino, Y. and Takasaki, M., Proposal of Force Measurement Using A Zero-Compliance Mechanism. Proceedings of XXI IMEKO World Congress "Measurement in Research and Industry" (2015), pp.317-320.

Mizuno, T., Ishino, Y. and Takasaki, M., Fabrication of a Three-Dimensional Force Measurement System using Double Series Magnetic Suspension. IFAC-PapersOnLine, Vol.49, No.21 (2016), pp.536-540.

Nahiyan, M. H. A., Mizuno, T., Takasaki, M., Ishino, Y., Hara, M. and Yamaguchi, D., Development of Three-Dimensional Force Measurement Instrument Using Zero-Compliance Mechanism. 29th Symposium on Electromagnetics and Dynamics (2017), pp.405-410.

Peris, J., Clijnen, J., Reynaerts, D., Brussel, H. V., Herijgers, P., Corteville, B. and Boone, S., A Micro Optical Force Sensor for Force Feedback During Minimally Invasive Robotic Surgery. Sensors and Actuators A, Vol.115 (2004), pp.447-455.

Sawada, H., Suenaga, H., Suzuki, T. and Ikeda, N., Status of MSBS Study at NAL. Proceedings of 2th International Symposium on Magnetic Suspension Technology, Part 1 (1994), pp.275-289.

Stefanescu, D. M., Handbook of Force Transducers (2011), Springer.

Surena, V., Bahareh, N. S. and Anahita, J., Atomic Force Microscopy Application in Biological Research: A Review Study. Iranian Journal of Medical Science, Vol.38, No.2 (2013), pp.76-83.

Woods, R. J. and Fearing, R. S., Flight Force Measurements for a Micromechanical Flying Insect. Proceedings of the IEEE/RSJ International Conference on Intelligent Robots and Systems, Vol.1 (2001), pp.355-362. 